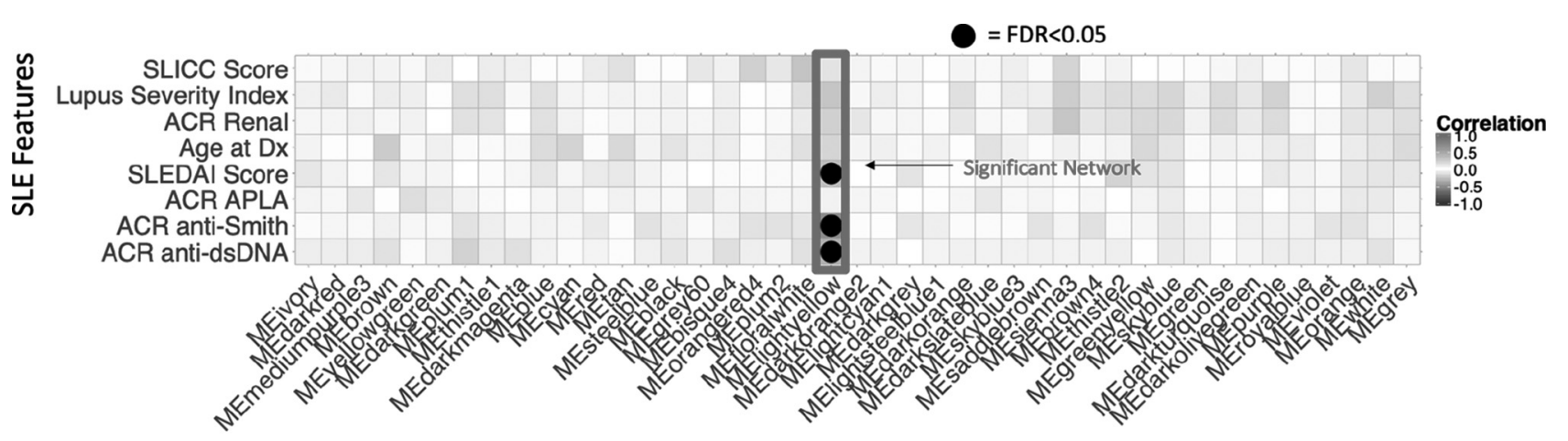

WGCNA Networks
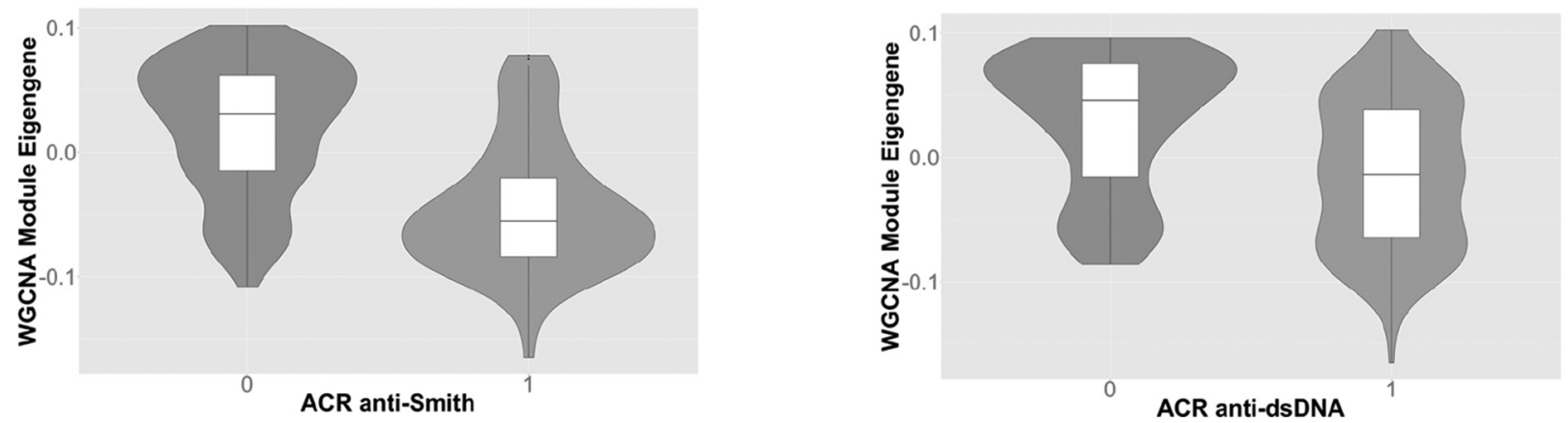

Abstract 149 Figure 1 Associations between WGCNA CpG network and SLE features. WGCNA was applied to methylation data and associated networks with relevant SLE clinical criteria. Top: Colors in squares represent Pearson correlations between individual phenotypes and module eigengenes with significant associations $(F D R<0.05)$ represented by a black circle. Bottom: Distribution of WGCNA eigengene for the identified network shows hypomethylation in patients with anti-Sm and anti-dsDNA serologies.

\section{ANTIMALARIAL AGENTS IMPROVE PHYSICAL FUNCTIONING IN PATIENTS WITH SYSTEMIC LUPUS ERYTHEMATOSUS}

Ioannis Parodis*, Sofia Soukka, Alvaro Gomez, Petter Johansson, Sharzad Emamikia, Yvonne Enman, Katerina Chatzidionysiou. Department of Medicine, Division of Rheumatology, Karolinska Institutet

\subsection{6/lupus-2019-Ism.150}

Background Patients with systemic lupus erythematosus (SLE) suffer an impaired health-related quality of life (HRQoL), and the majority of them experience fatigue as a major problem. Traditionally, treatment of SLE has been symptomatic, and antimalarial agents (AMA) are considered a cornerstone of SLE treatment. In previous literature, results regarding the effect of antimalarial agents on HRQoL have been conflicting. In this study, we aimed at investigating the potential influence of AMA on SLE patients self-perception of HRQoL aspects.

Methods We utilised pooled baseline data from the BLISS-52 and BLISS-76 clinical trials of belimumab $(n=1684)$. Access to data was granted by GlaxoSmithKline. The patients HRQoL and fatigue were self-reported using the Medical Outcomes Study (MOS) short form 36 (SF-36) health survey, the functional assessment of chronic illness therapy (FACIT)-Fatigue scale and the three-level EuroQol- 5 Dimension (EQ-5D) questionnaire. The non-parametric Mann-Whitney $U$ test was used for comparisons between AMA users and non-users. Linear regression models were next used in order to adjust for possible confounding factors; these included age, sex, ethnic origin, SLE disease activity, SLE duration, organ damage, corticosteroid use and use of other immunosuppressants.

Results Patients receiving AMA performed better than patients who did not receive AMA with regard to SF-36 physical component summary (PCS) scores $(\mathrm{p}=0.001)$, physical functioning $(p<0.001)$, role physical $(p=0.036)$, bodily pain $(p=0.016)$, FACIT-Fatigue scores $(p=0.046)$, EQ-5D score $(p=0.004)$ and EQ-5D visual analogue scale (VAS) scores $(p=0.001)$. However, only the difference regarding SF-36 physical functioning was found to be greater than the minimal clinically important difference (MCID) among all SF-36 and FACIT-Fatigue parameters (2.5 points for physical functioning). No MCID for EQ-5D scores or EQ-5D VAS scores has been validated. The observed association regarding physical functioning was still significant after adjustment for confounding factors. In this analysis, Asian patients reported better physical functioning while African/African American patients performed worse. High disease activity and organ damage were also independent factors of worse physical functioning, whereas corticosteroid use independently improved the outcome.

Conclusions AMA use contributes to better physical functioning in patients with SLE, independently of other factors.

Funding Source(s): The study was supported by grants from the Swedish Research Council, Professor Nanna Svartz Foundation (2017-00213), Swedish Rheumatism Association, King Gustaf Vs 80 year Foundation, Ingegerd Johanssons Fund, Stockholm County Council and Karolinska Institutet Foundations. 